\title{
Synthesis of Isoquinoline Derivatives Using ROM-RCM of Cyclobutene-Yne
}

\author{
Miwako Mori* ${ }^{\ddagger}$ Hideaki Wakamatsu, ${ }^{\dagger}$ Keisuke Tonogaki, ${ }^{\ddagger}$ Reiko Fujita, ${ }^{\dagger}$ \\ Tsuyoshi Kitamura, ${ }^{\ddagger}$ and Yoshihiro Sato, ${ }^{*}$
}

Graduate School of Pharmaceutical Sciences, Hokkaido University, Sapporo 060-0812, Japan and Tohoku Pharmaceutical University, 4-4-1 Komatsushima, Aoba-ku, Sendai 981-8558, Japan. mori@pharm.hokudai.ac.jp

\section{Supporting Information}

\section{Table of Contents}

- Experimental Section

S2-S10 


\section{Experimental Section.}

General Methods. Solvents were distilled under an argon atmosphere from sodium benzophenone (THF, Toluene) or $\mathrm{CaH}_{2}\left(\mathrm{CH}_{2} \mathrm{Cl}_{2}\right)$. All other reagents were purified when necessary by standard procedure. All reactions were conducted under an argon atmosphere unless it was described. ${ }^{1} \mathrm{H}$ NMR spectra were recorded at $400 \mathrm{MHz}$ or $270 \mathrm{MHz} .{ }^{13} \mathrm{C} \mathrm{NMR}$ spectra were recorded at $100 \mathrm{MHz}$ or $67.8 \mathrm{MHz} .{ }^{1} \mathrm{H}$ NMR and ${ }^{13} \mathrm{C}$ NMR shifts (ppm) were reported relative to internal tetramethylsilane $\left(\mathrm{Me}_{4} \mathrm{Si}\right)$. For ${ }^{13} \mathrm{C} \mathrm{NMR}$ spectra, carbon type is defined as $\left(\mathrm{CH}_{3}\right),\left(\mathrm{CH}_{2}\right)$, $(\mathrm{CH})$, or (C) on the basis of DEPT experiments. Kieselgel 60 (Merck, 70-230 mesh) and Kieselgel 60 (Merck, 230-400 mesh) were used for column chromatography or flash column chromatography, respectively.

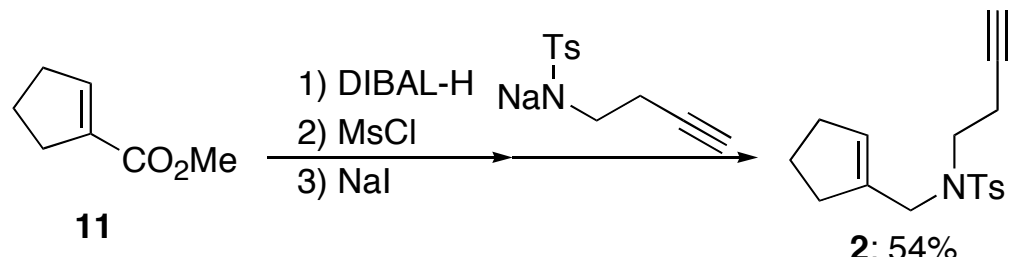

$N$-But-3-ynyl- $N$-cyclopent-1-enylmethyl-p-toluenesulfonamide (2).

To a solution of $11(0.64 \mathrm{~mL}, 5 \mathrm{mmol})$ in $\mathrm{CH}_{2} \mathrm{Cl}_{2}(12.5 \mathrm{~mL})$ was added DIBAL-H $(13 \mathrm{~mL}, 12.5$ mmol, $0.95 \mathrm{M}$ in hexane), and the solution was stirred at $-78{ }^{\circ} \mathrm{C}$ for $2 \mathrm{~h}$. To this solution was added $\mathrm{MeOH}$ and saturated Rochelle salt solution at $-78^{\circ} \mathrm{C}$ and the aqueous layer was extracted with $\mathrm{Et}_{2} \mathrm{O}$. The organic layer was washed with brine, dried over $\mathrm{Na}_{2} \mathrm{SO}_{4}$ and carefully concentrated to give crude alcohol (500 mg). To a solution of crude alcohol (500 mg, ca. $5 \mathrm{mmol})$ in ether (5 $\mathrm{mL})$ was added $\mathrm{Et}_{3} \mathrm{~N}(1.3 \mathrm{~mL}, 10 \mathrm{mmol})$, and $\mathrm{MsCl}(0.4 \mathrm{~mL}, 5 \mathrm{mmol})$ at $-78{ }^{\circ} \mathrm{C}$ and the solution was stirred at $0{ }^{\circ} \mathrm{C}$ for $2 \mathrm{~h}$. To this solution was added $\mathrm{NaI}(924 \mathrm{mg}, 6.1 \mathrm{mmol})$ at $0{ }^{\circ} \mathrm{C}$ and the solution was stirred at $\mathrm{rt}$ for $3 \mathrm{~h}$. The resulting solution (cyclopentenyl iodide in ether) was used for the next reaction without further purification. To a suspension of $\mathrm{NaH}$ (244 mg, $6 \mathrm{mmol}, 60 \%$ oil 
suspension) in DMF (5 mL) was added a solution of $\mathbf{1 3}^{1}(1.1 \mathrm{~g}, 5 \mathrm{mmol})$ in DMF $(5 \mathrm{~mL})$ at $0{ }^{\circ} \mathrm{C}$, and the solution was stirred for $30 \mathrm{~min}$ at $0{ }^{\circ} \mathrm{C}$. To this solution was added cyclopentenyl iodide in ether. The whole solution was stirred at $\mathrm{rt}$ for $15 \mathrm{~h}$. To this solution was added saturated $\mathrm{NH}_{4} \mathrm{Cl}$ solution, and the aqueous layer was extracted with AcOEt. The organic layer was washed with brine, dried over $\mathrm{Na}_{2} \mathrm{SO}_{4}$, and concentrated. The residue was purified by column chromatography on silica gel (Hexane/AcOEt, 5:1) to yield 2 (800 mg, 53\%, 4 steps) as colorless needles: $\mathrm{mp} 50-51{ }^{\circ} \mathrm{C}$ (Hexane); IR (nujol) 3282, 2924, 2851, 1654, 1598, 1326, $1153 \mathrm{~cm}^{-1} ;{ }^{1} \mathrm{H}$ NMR (400 MHz, CDCl 3 ) $\delta 1.85(\mathrm{~m}, 2 \mathrm{H}), 1.96(\mathrm{t}, J=2.4 \mathrm{~Hz}, 1 \mathrm{H}), 2.16(\mathrm{~m}, 2 \mathrm{H}), 2.30(\mathrm{~m}, 2 \mathrm{H}), 2.40-2.44(\mathrm{~m}, 2 \mathrm{H}), 2.43$ (s, $3 \mathrm{H}), 3.26(\mathrm{~m}, 2 \mathrm{H}), 3.87$ (s, $2 \mathrm{H}), 5.57(\mathrm{~s}, 1 \mathrm{H}), 7.30$ (d, J = 8.0 Hz, $2 \mathrm{H}), 7.70(\mathrm{~d}, J=8.0 \mathrm{~Hz}, 2 \mathrm{H})$; ${ }^{13} \mathrm{C}$ NMR $\left(100 \mathrm{MHz}, \mathrm{CDCl}_{3}\right) \delta 18.9\left(\mathrm{CH}_{2}\right), 21.2\left(\mathrm{CH}_{3}\right), 23.2\left(\mathrm{CH}_{2}\right), 32.2\left(\mathrm{CH}_{2}\right), 32.9\left(\mathrm{CH}_{2}\right), 46.2$ $\left(\mathrm{CH}_{2}\right), 48.7\left(\mathrm{CH}_{2}\right), 69.9(\mathrm{CH}), 80.8(\mathrm{C}), 126.7(\mathrm{CH}$ x 2), $129.3(\mathrm{CH}$ x 2), $129.5(\mathrm{CH}), 136.5(\mathrm{C})$, 139.0 (C), 142.9 (C); LRMS m/z $303\left(\mathrm{M}^{+}\right), 264,184,155,148,91,81$; Anal. calcd for $\mathrm{C}_{17} \mathrm{H}_{21} \mathrm{NO}_{2} \mathrm{~S}: \mathrm{C}, 67.29 ; \mathrm{H}, 6.98 ; \mathrm{N}, 4.62 ; \mathrm{S}, 10.57$. Found: C, 67.12; H, 6.99; N, 4.48; S, 10.42.

${ }^{1}$ a) Short, K. M.; Ziegler, C. B. Jr. Tetrahedron Lett. 1995, 36, 355. b) Henry, J. R.; Marcin, L. R.; McIntosh, M. C.; Scola, P. M.; Harris, D. Jr.; Weinreb, S. M. Tetrahedron Lett. 1989, 30, 5709. 


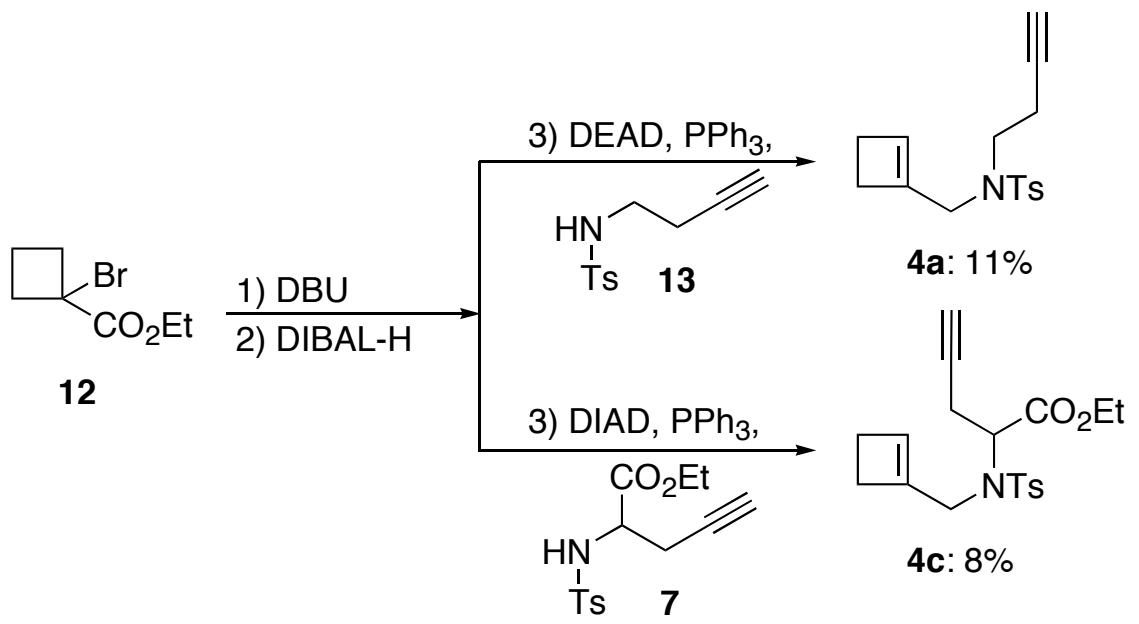

\section{$N$-But-3-ynyl- $N$-cyclobut-1-enylmethyl-p-toluenesulfonamide (4a).}

A mixture of $12(2.0 \mathrm{~mL}, 12.35 \mathrm{mmol})$ and DBU $(2.8 \mathrm{~mL}, 18.53 \mathrm{mmol})$ was stirred for $1 \mathrm{~h}$ at 90 ${ }^{\circ} \mathrm{C}$. To the resulting mixture was added $\mathrm{CH}_{2} \mathrm{Cl}_{2}(10 \mathrm{~mL})$. The organic phase was washed with $10 \% \mathrm{HCl}$ solution and dried over $\mathrm{MgSO}_{4}$. After filtration, volatiles were removed carefully under reduce pressure $\left(360 \mathrm{mmHg}, \sim 20{ }^{\circ} \mathrm{C}\right)$. The residue was dissolved in dry $\mathrm{CH}_{2} \mathrm{Cl}_{2}(15 \mathrm{~mL})$ and cooled to $-78{ }^{\circ} \mathrm{C}$. DIBAL-H (39.5 mL, $37.06 \mathrm{mmol}, 0.94 \mathrm{M}$ in hexane) was added to a solution and the whole solution was stirred at the same temperature for $1.5 \mathrm{~h}$. After the spot of the starting material had disappeared on $\mathrm{TLC}, \mathrm{HCO}_{2} \mathrm{Me}(1.0 \mathrm{~mL})$ and saturated Rochelle salt solution $(20 \mathrm{~mL})$ were added to the solution. The resulting turbidity mixture was continuously stirred until turn to clear solution. The solution was extracted with $\mathrm{Et}_{2} \mathrm{O}$ and the organic phase was dried over $\mathrm{MgSO}_{4}$. After filtration, volatiles were removed carefully under reduce pressure $\left(400 \mathrm{mmHg}, \sim 30{ }^{\circ} \mathrm{C}\right)$. $\operatorname{DEAD}\left(3.1 \mathrm{~mL}, 6.80 \mathrm{mmol}, 40 \%\right.$ in Toluene) was added to the solution of the crude product, $\mathbf{1 3}^{1}$ $(1.38 \mathrm{~g}, 6.18 \mathrm{mmol})$ and $\mathrm{PPh}_{3}(1.78 \mathrm{~g}, 6.80 \mathrm{mmol})$ in $\mathrm{THF}(30 \mathrm{~mL})$ at $0{ }^{\circ} \mathrm{C}$. The solution was stirred at $\mathrm{rt}$ for $14 \mathrm{~h}$ and then concentrated. The residue was purified by column chromatography on silica gel (Hexane/AcOEt, 3:1 and Hexane/ $\left.\mathrm{Et}_{2} \mathrm{O}, 5: 1\right)$ to afford $\mathbf{4 a}(376.6 \mathrm{mg}, 11 \%$ from 12) as a colorless liquid and recovered 13 (0.98 g): IR (neat) 2120 (w), 1735 (m), 1598 (m), 1342 (s), 1158 (s) $\mathrm{cm}^{-1} ;{ }^{1} \mathrm{H}$ NMR $\left(270 \mathrm{MHz}, \mathrm{CDCl}_{3}\right) \delta 1.98(\mathrm{dd}, J=2.4,3.0 \mathrm{~Hz}, 1 \mathrm{H}), 2.23-2.32(\mathrm{~m}, 4 \mathrm{H}), 2.42$ (s, $3 \mathrm{H}), 2.42-2.50(\mathrm{~m}, 2 \mathrm{H}), 3.33(\mathrm{t}, J=7.6 \mathrm{~Hz}, 2 \mathrm{H}), 3.81(\mathrm{~s}, 2 \mathrm{H}), 5.82(\mathrm{~s}, 1 \mathrm{H}), 7.29(\mathrm{~d}, J=8.5 \mathrm{~Hz}$, 
$2 \mathrm{H}), 7.70(\mathrm{~d}, J=8.5 \mathrm{~Hz}, 2 \mathrm{H})$; EI-LRMS $m / z 289\left(\mathrm{M}^{+}\right), 250,184,155,134,91 ;{ }^{13} \mathrm{C}$ NMR $(67.8$ $\left.\mathrm{MHz}, \mathrm{CDCl}_{3}\right) \delta 19.2\left(\mathrm{CH}_{2}\right), 21.4\left(\mathrm{CH}_{3}\right), 26.7\left(\mathrm{CH}_{2}\right), 30.4\left(\mathrm{CH}_{2}\right), 46.4\left(\mathrm{CH}_{2}\right), 47.9\left(\mathrm{CH}_{2}\right), 70.1$ $(\mathrm{CH}), 81.0$ (C), 127.1 (CH x 2), 129.5 (CH x 2), 132.9 (CH), 136.9 (C), 143.3 (C), 143.6 (C); EIHRMS $m / z$ calcd for $\mathrm{C}_{16} \mathrm{H}_{19} \mathrm{O}_{2} \mathrm{NS}\left(\mathrm{M}^{+}\right)$289.1137, found 289.1141.

Ethyl 2-[cyclobut-1-enylmethyl-( $p$-toluenesulfonyl)-amino]-pent-4-ynoate (4c).

In a similar manner to that for the synthesis of $\mathbf{4 a}$ from $\mathbf{1 2}, \mathbf{4 c}(176.6 \mathrm{mg}, 8 \% \mathrm{~d})$ was synthesized from 12 (1.0 mL, $6.18 \mathrm{mmol})$ and $7^{2}(359.3 \mathrm{mg}, 1.22 \mathrm{mmol})$ : IR (neat) $2124(\mathrm{w}), 1732(\mathrm{~s}), 1598$ (m), 1337 (s), $1164(\mathrm{~s}) \mathrm{cm}^{-1} ;{ }^{1} \mathrm{H}$ NMR (400 MHz, $\left.\mathrm{CDCl}_{3}\right) \delta 1.18(\mathrm{t}, J=7.2 \mathrm{~Hz}, 3 \mathrm{H}), 2.01(\mathrm{t}, J=$ $2.6 \mathrm{~Hz}, 1 \mathrm{H}), 2.25-2.40(\mathrm{~m}, 4 \mathrm{H}), 2.42$ (s, $3 \mathrm{H}), 2.72$ (ddd, $J=2.6,8.2,17.0 \mathrm{~Hz}, 1 \mathrm{H}), 2.88$ (ddd, $J=$ 2.6, 6.5, $17.0 \mathrm{~Hz}, 1 \mathrm{H}), 3.77(\mathrm{~d}, J=17.0 \mathrm{~Hz}, 1 \mathrm{H}), 3.91(\mathrm{~d}, J=17.0 \mathrm{~Hz}, 1 \mathrm{H}), 4.00-4.13(\mathrm{~m}, 2 \mathrm{H})$, $4.72(\mathrm{dd}, J=6.5,8.2 \mathrm{~Hz}, 1 \mathrm{H}), 5.89(\mathrm{~s}, 1 \mathrm{H}), 7.27(\mathrm{~d}, J=8.2 \mathrm{~Hz}, 2 \mathrm{H}), 7.76(\mathrm{~d}, J=8.2 \mathrm{~Hz}, 2 \mathrm{H})$; ${ }^{13} \mathrm{C}$ NMR $\left(100 \mathrm{MHz}, \mathrm{CDCl}_{3}\right) \delta 14.0\left(\mathrm{CH}_{3}\right), 21.2\left(\mathrm{CH}_{2}\right), 21.6\left(\mathrm{CH}_{3}\right), 26.7\left(\mathrm{CH}_{2}\right), 30.5\left(\mathrm{CH}_{2}\right), 45.6$ $\left(\mathrm{CH}_{2}\right), 58.7(\mathrm{CH}), 61.6\left(\mathrm{CH}_{2}\right), 71.2(\mathrm{CH}), 79.6(\mathrm{C}), 127.6(\mathrm{CH} \times 2), 129.2(\mathrm{CH} \times 2), 132.6(\mathrm{CH})$, 137.2 (C), 143.3 (C), 144.5 (C), 169.1 (C); EI-LRMS m/z 362 (M+1), 322, 288, 256, 222, 206, $155,105,91$; EI-HRMS $m / z$ calcd for $\mathrm{C}_{19} \mathrm{H}_{23} \mathrm{O}_{4} \mathrm{NS}\left(\mathrm{M}^{+}\right) 361.1348$, found 361.1373 .

\footnotetext{
2 a) Kotha, S.; Sreenivasachary, N. Chem. Commun. 2000, 503. b) Kotha, S.; Sreenivasachary, N. Eur. J. Org. Chem. 2001, 3375.
} 


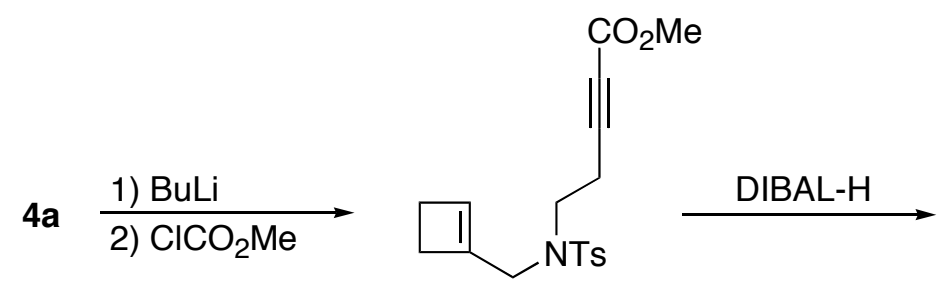

14: $68 \%$

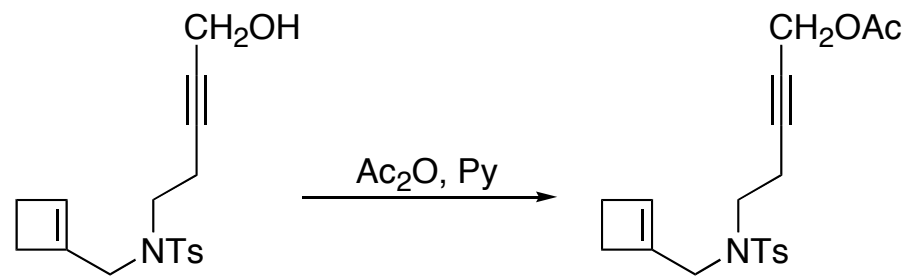

15: $98 \%$

4b: $87 \%$

Methyl 5-[Cyclobut-1-enylmethyl-( $p$-toluenesulfonyl)-amino]-pent-2-ynoate (14).

To a solution of $\mathbf{4 a}(320.2 \mathrm{mg}, 1.11 \mathrm{mmol})$ in THF $(11 \mathrm{~mL})$ was added BuLi $(0.83 \mathrm{~mL}, 1.33 \mathrm{mmol}$, $1.60 \mathrm{M}$ in hexane) at $-78^{\circ} \mathrm{C}$. After stirring at the same temperature for $0.5 \mathrm{~h}$, freshly distilled $\mathrm{ClCO}_{2} \mathrm{Me}(0.17 \mathrm{~mL}, 2.21 \mathrm{mmol})$ was added and the solution was stirred for $1 \mathrm{~h}$. Saturated $\mathrm{NH}_{4} \mathrm{Cl}$ solution was added and the aqueous layer was extracted with AcOEt. The organic phase was washed with brine, dried over $\mathrm{MgSO}_{4}$ and concentrated. The residue was purified by column chromatography on silica gel (Hexane/Et $2 \mathrm{O}, 5: 1)$ to afford $14(260.1 \mathrm{mg}, 68 \%)$ as a colorless liquid and recovered 4a (38.0 mg, 12\%): IR (neat) 2242 (s), 1717 (s), 1598 (m), 1345 (s), 1260 (s), 1159 (s) $\mathrm{cm}^{-1} ;{ }^{1} \mathrm{H}$ NMR $\left(270 \mathrm{MHz}, \mathrm{CDCl}_{3}\right) \delta$ 2.24-2.28 (m, $\left.2 \mathrm{H}\right), 2.28-2.33(\mathrm{~m}, 2 \mathrm{H}), 2.43(\mathrm{~s}, 3 \mathrm{H}), 2.64$ (t, $J=7.5 \mathrm{~Hz}, 2 \mathrm{H}), 3.36(\mathrm{t}, J=7.5 \mathrm{~Hz}, 2 \mathrm{H}), 3.76$ (s, $3 \mathrm{H}), 3.81$ (br, $2 \mathrm{H}), 5.84(\mathrm{br}, 1 \mathrm{H}), 7.30$ (d, $J$ $=8.4 \mathrm{~Hz}, 2 \mathrm{H}), 7.70(\mathrm{~d}, J=8.4 \mathrm{~Hz}, 2 \mathrm{H}) ;{ }^{13} \mathrm{C} \mathrm{NMR}\left(67.8 \mathrm{MHz}, \mathrm{CDCl}_{3}\right) \delta 19.3\left(\mathrm{CH}_{2}\right), 21.1\left(\mathrm{CH}_{3}\right)$, $26.5\left(\mathrm{CH}_{2}\right), 30.1\left(\mathrm{CH}_{2}\right), 45.2\left(\mathrm{CH}_{2}\right), 47.9\left(\mathrm{CH}_{2}\right), 52.3\left(\mathrm{CH}_{3}\right), 73.9(\mathrm{C}), 85.6(\mathrm{C}), 126.9(\mathrm{CH}$ x 2), 129.4 (CH x 2), 132.9 (CH), 136.3 (C), 143.1 (C), 143.2 (C), 153.4 (C); EI-LRMS m/z $347\left(\mathrm{M}^{+}\right)$, 316, 294, 250, 192, 184, 155, 91; EI-HRMS $m / z$ calcd for $\mathrm{C}_{18} \mathrm{H}_{21} \mathrm{O}_{4} \mathrm{NS}\left(\mathrm{M}^{+}\right)$347.1191, found 347.1197.

$N$-Cyclobut-1-enylmethyl- $N$-(5-hydroxy-pent-3-ynyl)-p-toluenesulfonamide (15). 
To a solution of $14(334.3 \mathrm{mg}, 0.96 \mathrm{mmol})$ in toluene $(10 \mathrm{~mL})$ was added DIBAL-H $(4.2 \mathrm{~mL}, 4.23$ mmol, 1.01 $\mathrm{M}$ in toluene) at $-25^{\circ} \mathrm{C}$. The solution was stirred at the same temperature for $1.5 \mathrm{~h}$. AcOEt $(5.0 \mathrm{~mL})$ and saturated Rochelle salt solution $(20 \mathrm{~mL})$ were added to this solution. The resulting turbidity mixture was continuously stirred until turn to clear solution. The solution was extracted with AcOEt and the organic phase was washed with brine, dried over $\mathrm{MgSO}_{4}$ and concentrated. The residue was purified by column chromatography on silica gel (Hexane/AcOEt, 3:2) to afford 15 (300.0 mg, 98\%) as a colorless liquid: IR (neat) 3515 (m), 2226 (w), 1598 (m), 1341 (s), 1158 (s) cm ${ }^{-1} ;{ }^{1} \mathrm{H}$ NMR (270 MHz, $\mathrm{CDCl}_{3}$ ) $\delta 2.16$ (br, $\left.1 \mathrm{H}\right), 2.21-2.31$ (m, $\left.4 \mathrm{H}\right), 2.42$ (s, $3 \mathrm{H}), 2.44-2.53(\mathrm{~m}, 2 \mathrm{H}), 3.32(\mathrm{t}, J=7.5 \mathrm{~Hz}, 2 \mathrm{H}), 3.80(\mathrm{br}, 2 \mathrm{H}), 4.21(\mathrm{t}, J=2.1 \mathrm{~Hz}, 2 \mathrm{H}), 5.81$ (br, $1 \mathrm{H}), 7.29(\mathrm{~d}, J=8.2 \mathrm{~Hz}, 2 \mathrm{H}), 7.70(\mathrm{~d}, J=8.2 \mathrm{~Hz}, 2 \mathrm{H}) ;{ }^{13} \mathrm{C} \mathrm{NMR}\left(67.8 \mathrm{MHz}, \mathrm{CDCl}_{3}\right) \delta 19.3$ $\left(\mathrm{CH}_{2}\right), 21.4\left(\mathrm{CH}_{3}\right), 26.7\left(\mathrm{CH}_{2}\right), 30.3\left(\mathrm{CH}_{2}\right), 46.3\left(\mathrm{CH}_{2}\right), 47.6\left(\mathrm{CH}_{2}\right), 50.9\left(\mathrm{CH}_{2}\right), 80.3(\mathrm{C}), 82.4$ (C), $127.0(\mathrm{CH} \times 2), 129.5(\mathrm{CH} \times 2), 132.8(\mathrm{CH}), 136.8(\mathrm{C}), 143.2$ (C), 143.5 (C); EI-LRMS m/z $319\left(\mathrm{M}^{+}\right), 250,184,155,91$; EI-HRMS $\mathrm{m} / \mathrm{z}$ calcd for $\mathrm{C}_{17} \mathrm{H}_{21} \mathrm{O}_{3} \mathrm{NS}\left(\mathrm{M}^{+}\right)$319.1242, found 319.1233.

\section{5-[Cyclobut-1-enylmethyl-(p-toluenesulfonyl)-amino]-pent-2-ynyl acetate (4b).}

To a solution of $15(110.7 \mathrm{mg}, 0.35 \mathrm{mmol})$ and pyridine $(0.28 \mathrm{~mL}, 3.47 \mathrm{mmol})$ in $\mathrm{CH}_{2} \mathrm{Cl}_{2}(4 \mathrm{~mL})$ was added $\mathrm{Ac}_{2} \mathrm{O}(0.16 \mathrm{~mL}, 1.73 \mathrm{mmol})$ at $0{ }^{\circ} \mathrm{C}$. The solution was stirred at $0{ }^{\circ} \mathrm{C}$ for $0.5 \mathrm{~h}$ and at $\mathrm{rt}$ for $2 \mathrm{~h}$. The solution was poured onto ice, and the aqueous layer was extracted with AcOEt. The organic phase was washed with brine, dried over $\mathrm{MgSO}_{4}$ and concentrated. The residue was purified by column chromatography on silica gel (Hexane/AcOEt, 2:1) to afford $\mathbf{4 b}$ (108.4 mg, 87\%) as a colorless liquid: IR (neat) 2240 (w), 1748 (s), 1598 (w), 1344 (s), 1227 (s), 1159 (s) cm

${ }^{1} ;{ }^{1} \mathrm{H}$ NMR $\left(270 \mathrm{MHz}, \mathrm{CDCl}_{3}\right.$ ) $\delta 2.09$ (s, $\left.3 \mathrm{H}\right), 2.23-2.32$ (m, $\left.4 \mathrm{H}\right), 2.42$ (s, $\left.3 \mathrm{H}\right), 2.47-2.55$ (m, 2 H), $3.31(\mathrm{t}, J=7.6 \mathrm{~Hz}, 2 \mathrm{H}), 3.80(\mathrm{br}, 2 \mathrm{H}), 4.62(\mathrm{t}, J=2.1 \mathrm{~Hz}, 2 \mathrm{H}), 5.81(\mathrm{br}, 1 \mathrm{H}), 7.29(\mathrm{~d}, J=8.2$ $\mathrm{Hz}, 2 \mathrm{H}), 7.70(\mathrm{~d}, J=8.2 \mathrm{~Hz}, 2 \mathrm{H}) ;{ }^{13} \mathrm{C} \mathrm{NMR}\left(67.8 \mathrm{MHz}, \mathrm{CDCl}_{3}\right) \delta 19.4\left(\mathrm{CH}_{2}\right), 20.6\left(\mathrm{CH}_{3}\right), 21.3$ $\left(\mathrm{CH}_{3}\right), 26.6\left(\mathrm{CH}_{2}\right), 30.2\left(\mathrm{CH}_{2}\right), 46.2\left(\mathrm{CH}_{2}\right), 47.8\left(\mathrm{CH}_{2}\right), 52.3\left(\mathrm{CH}_{2}\right), 75.7(\mathrm{C}), 83.8(\mathrm{C}), 127.0(\mathrm{CH}$ x 2), 129.5 (CH x 2), 132.7 (CH), 136.9 (C), 143.2 (C), 143.5 (C), 170.1 (C); EI-LRMS m/z 361 
$\left(\mathrm{M}^{+}\right), 302,250,184,155,91$; EI-HRMS $m / z$ calcd for $\mathrm{C}_{19} \mathrm{H}_{23} \mathrm{O}_{4} \mathrm{NS}\left(\mathrm{M}^{+}\right)$361.1348, found 361.1350 .

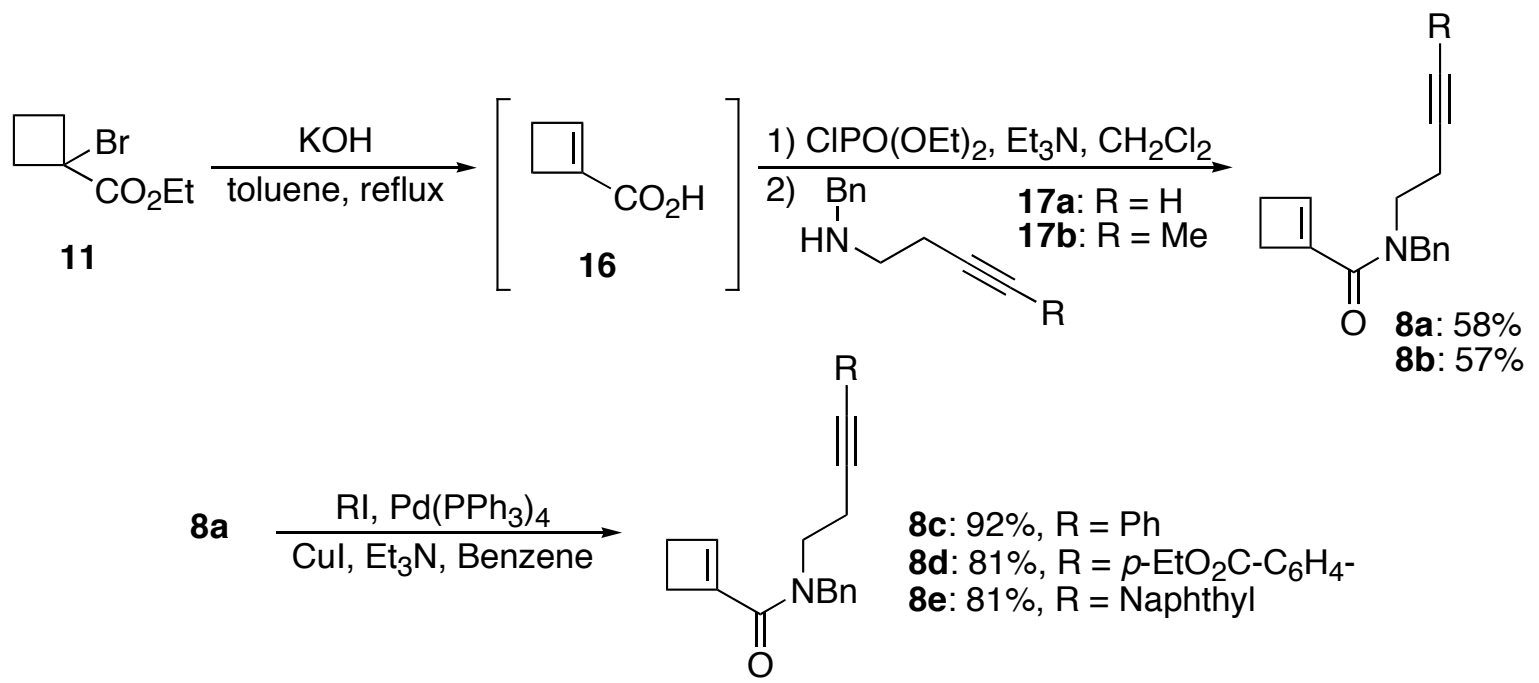

\section{$N$-Benzyl- $N$-(but-3-ynyl)-1-cyclobutene-1-carboxamide (8a).}

To a solution of $\mathrm{KOH}(1.46 \mathrm{~g}, 25.94 \mathrm{mmol})$ in toluene $(25 \mathrm{~mL})$ was added $11(1.0 \mathrm{~mL}, 6.18 \mathrm{~mL})$ and the solution was refluxed for $45 \mathrm{~min}$. After cooling, water was added and an aqueous layer was washed with petroleum ether. An aqueous layer was acidified by addition of $10 \% \mathrm{HCl}$. The acidic phase was extracted with ether and the organic phase was washed with brine, dried over $\mathrm{MgSO}_{4}$ and concentrated to afford crude 1-cyclobutenecarboxylic acid. To a solution of crude 1cyclobutenecarboxylic acid $(6.18 \mathrm{mmol})$ in $\mathrm{CH}_{2} \mathrm{Cl}_{2}(21 \mathrm{~mL})$ was added $\mathrm{Et}_{3} \mathrm{~N}(1.7 \mathrm{~mL}, 12.35$ mmol) and $\mathrm{ClPO}(\mathrm{OEt})_{2}(1.1 \mathrm{~mL}, 7.41 \mathrm{mmol})$ at $0{ }^{\circ} \mathrm{C}$. After stirring for $1 \mathrm{~h}$ at the same temperature, $17 \mathbf{a}^{3}(1.28 \mathrm{~g}, 8.03 \mathrm{mmol})$ in $\mathrm{CH}_{2} \mathrm{Cl}_{2}(10 \mathrm{~mL})$ was added. The resulting solution was stirred at $\mathrm{rt}$ for $2 \mathrm{~h}$. Saturated $\mathrm{NaHCO}_{3}$ solution was added, and the aqueous phase was extracted with AcOEt. The combined organic phase was washed with saturated $\mathrm{NH}_{4} \mathrm{Cl}$ solution and brine, dried over $\mathrm{MgSO}_{4}$, filtered, and concentrated. The residue was purified by column chromatography on silica

\footnotetext{
${ }^{3}$ Mengelberg, M. Chem. Ber. 1956, 89, 1185.
} 
gel (Hexane/AcOEt, 2:1) to afford $\mathbf{8 a}(853.4 \mathrm{mg}, 58 \%, 2$ steps) as a pale yellow liquid: IR (neat) 2119 (w), 1696 (s), 1631 (s), 1422 (s), 1243 (s) cm ${ }^{-1} ;{ }^{1} \mathrm{H}$ NMR (270 MHz, DMSO, $\left.100{ }^{\circ} \mathrm{C}\right) \delta$ 2.38-2.44 (m, 4 H), 2.65 (t, J=2.5 Hz, 1 H), 2.74 (m, 2 H), 3.48 (t, J = 7.1 Hz, 2 H), 4.69 (s, 2 H), 6.40 (br, $1 \mathrm{H})$, 7.22-7.37 (m, $5 \mathrm{H}$ ); EI-LRMS m/z $239\left(\mathrm{M}^{+}\right), 200,142$, 91; EI-HRMS m/z calcd for $\mathrm{C}_{16} \mathrm{H}_{17} \mathrm{ON}\left(\mathrm{M}^{+}\right)$239.1310, found 239.1302.

\section{$N$-Benzyl- $N$-(pent-3-ynyl)-1-cyclobutene-1-carboxamide (8b).}

In a similar manner to that for the synthesis of $\mathbf{8 a}$ from $\mathbf{1 2}, \mathbf{8 b}(448.9 \mathrm{mg}, 57 \%, 2$ steps) was synthesized from $12(0.5 \mathrm{~mL}, 3.09 \mathrm{mmol})$ and $\mathbf{1 7} \mathbf{b}^{4}(695.6 \mathrm{mg}, 4.01 \mathrm{mmol})$ : IR (neat) 1627 (s), 1586 (s), $1422(\mathrm{~m}) \mathrm{cm}^{-1} ;{ }^{1} \mathrm{H}$ NMR $\left(270 \mathrm{MHz}, \mathrm{DMSO}, 100{ }^{\circ} \mathrm{C}\right) \delta 1.71(\mathrm{t}, J=2.6 \mathrm{~Hz}, 3 \mathrm{H}), 2.33$ (tq, $J=7.1,2.6 \mathrm{~Hz}, 2 \mathrm{H}), 2.39-2.43(\mathrm{~m}, 2 \mathrm{H}), 2.75$ (t, $J=3.2 \mathrm{~Hz}, 2 \mathrm{H}), 3.44(\mathrm{t}, J=7.1 \mathrm{~Hz}, 2 \mathrm{H}), 4.67$ (s, 2 H), 6.40 (brs, 1 H), 7.21-7.37 (m, 5 H); EI-LRMS m/z $253\left(\mathrm{M}^{+}\right), 238,200,156,120$, 91; EIHRMS $m / z$ calcd for $\mathrm{C}_{17} \mathrm{H}_{19} \mathrm{ON}\left(\mathrm{M}^{+}\right)$253.1467, found 253.1486.

\section{$N$-Benzyl- $N$-(4-phenyl-but-3-ynyl)-1-cyclobutene-1-carboxamide (8c).}

To a suspension of $\mathrm{Pd}\left(\mathrm{PPh}_{3}\right)_{4}(45.2 \mathrm{mg}, 39.15 \mu \mathrm{mol})$ and $\mathrm{CuI}(7.5 \mathrm{mg}, 39.15 \mu \mathrm{mol})$ in $\mathrm{Et}_{3} \mathrm{~N}(2$ $\mathrm{mL}$ ) was added $\mathrm{PhI}(0.09 \mathrm{~mL}, 0.82 \mathrm{mmol})$ at rt. After stirred for $10 \mathrm{~min}, \mathbf{8 a}(187.4 \mathrm{mg}, 0.78 \mathrm{mmol})$ in benzene $(1 \mathrm{~mL})$ was added to this solution, and the resulting solution was stirred at $\mathrm{rt}$ for $16 \mathrm{~h}$ and concentrated. The residue was purified by column chromatography on silica gel (Hexane/AcOEt, 2:1) to afford 8c (226.1 mg, 92\%) as a pale yellow liquid: IR (neat) 1713 (s), 1628 (s), 1586 (s), 1492 (m), 1421 (s) cm ${ }^{-1} ;{ }^{1} \mathrm{H}$ NMR (270 MHz, DMSO, $\left.100{ }^{\circ} \mathrm{C}\right) \delta$ 2.38-2.42 (br, $2 \mathrm{H}), 2.48$ (t, $J=6.9 \mathrm{~Hz}, 2 \mathrm{H}), 2.74-2.78(\mathrm{br}, 2 \mathrm{H}), 3.60$ (t, $J=6.9 \mathrm{~Hz}, 2 \mathrm{H}), 4.74$ (s, $2 \mathrm{H}), 6.44$ (brs, $1 \mathrm{H}), 7.23-7.38(\mathrm{~m}, 10 \mathrm{H})$; EI-LRMS $\mathrm{m} / z 315\left(\mathrm{M}^{+}\right), 200,91$; EI-HRMS $\mathrm{m} / z$ calcd for $\mathrm{C}_{22} \mathrm{H}_{21} \mathrm{ON}$ $\left(\mathrm{M}^{+}\right)$315.1623, found 315.1624.

\footnotetext{
${ }^{4}$ Van Houten, K. A.; Kim, J.-M.; Bogdan, M. A.; Ferri, D. C.; Mariano, P. S. J. Am. Chem. Soc. 1998, 120, 5864.
} 
Ethyl 4-\{4-[benzyl-(cyclobut-1-enecarbonyl)-amino]-but-1-ynyl\}-benzoate (8d).

In a similar manner to that for the synthesis of 8c from 8a, 8d (203.0 mg, 81\%) was synthesized from $8 \mathbf{a}(154.7 \mathrm{mg}, 0.65 \mathrm{mmol})$ and Ethyl 4-iodobenzoate $(0.11 \mathrm{~mL}, 0.68 \mathrm{mmol})$ : $\mathrm{IR}$ (neat) 2231 (w), 1716 (s), 1627 (s), 1607 (s), 1584 (m), 1274 (s), 755 (s) cm ${ }^{-1} ;{ }^{1} \mathrm{H}$ NMR (270 MHz, DMSO, $\left.100{ }^{\circ} \mathrm{C}\right) \delta 1.33(\mathrm{t}, J=7.1 \mathrm{~Hz}, 3 \mathrm{H}), 2.38-2.42(\mathrm{~m}, 2 \mathrm{H}), 2.71(\mathrm{t}, J=6.9 \mathrm{~Hz}, 2 \mathrm{H}), 2.76(\mathrm{t}, J=3.2 \mathrm{~Hz}$, $2 \mathrm{H}), 3.61(\mathrm{t}, J=6.9 \mathrm{~Hz}, 2 \mathrm{H}), 4.33(\mathrm{q}, J=7.1 \mathrm{~Hz}, 2 \mathrm{H}), 4.74(\mathrm{~s}, 2 \mathrm{H}), 6.44(\mathrm{br}, 1 \mathrm{H}), 7.24-7.38$ (m, $5 \mathrm{H}), 7.47$ (d, $J=8.6 \mathrm{~Hz}, 2 \mathrm{H}), 7.91$ (d, $J=8.6 \mathrm{~Hz}, 2 \mathrm{H})$; EI-LRMS m/z $387\left(\mathrm{M}^{+}\right), 342$, 200, 91; EI-HRMS $m / z$ calcd for $\mathrm{C}_{25} \mathrm{H}_{25} \mathrm{O}_{3} \mathrm{~N}\left(\mathrm{M}^{+}\right)$387.1834, found 387.1826.

\section{$N$-Benzyl- $N$-(4-naphthalen-1-yl-but-3-ynyl)-1-cyclobut-1-enecarboxamide (8e).}

In a similar manner to that for the synthesis of $\mathbf{8 c}$ from $\mathbf{8 a}, \mathbf{8 e}(213.3 \mathrm{mg}, 81 \%)$ was synthesized from $8 \mathbf{a}(172.0 \mathrm{mg}, 0.72 \mathrm{mmol})$ and 1-Iodonaphthalene (0.11 mL, $0.75 \mathrm{mmol})$ : IR (neat) $2224(\mathrm{w})$, 1718 (w), 1625 (s), 1585 (m), 1421 (s), 755 (s) cm ${ }^{-1} ;{ }^{1} \mathrm{H}$ NMR (270 MHz, DMSO, $\left.100{ }^{\circ} \mathrm{C}\right) \delta 2.39$ (br, $2 \mathrm{H}), 2.77$ (br, $2 \mathrm{H}), 2.85(\mathrm{t}, J=6.8 \mathrm{~Hz}, 2 \mathrm{H}), 3.65-3.75(\mathrm{~m}, 2 \mathrm{H}), 4.79$ (s, $2 \mathrm{H}), 6.46$ (br, $1 \mathrm{H})$, 7.25-8.25 (m, $12 \mathrm{H})$; EI-LRMS m/z $364\left(\mathrm{M}^{+}-1\right), 200,178,165,91$; EI-HRMS $m / z$ calcd for $\mathrm{C}_{26} \mathrm{H}_{23} \mathrm{ON}\left(\mathrm{M}^{+}\right)$365.1779, found 365.1770. 